\title{
Spatial variation of insecticide resistance in the dengue vector Aedes aegypti presents unique vector control challenges
}

Regan Deming ${ }^{1}$, Pablo Manrique-Saide², Anuar Medina Barreiro², Edgar Ulises Koyoc Cardeña², Azael Che-Mendoza ${ }^{3}$, Bryant Jones ${ }^{1}$, Kelly Liebmann ${ }^{4}$, Lucrecia Vizcaino ${ }^{4}$, Gonzalo Vazquez-Prokopec ${ }^{1}$ and Audrey Lenhart ${ }^{4 *}$

\begin{abstract}
Background: Dengue is a major public health problem in Mexico, where the use of chemical insecticides to control the principal dengue vector, Aedes aegypti, is widespread. Resistance to insecticides has been reported in multiple sites, and the frequency of $k d r$ mutations associated with pyrethroid resistance has increased rapidly in recent years. In the present study, we characterized patterns of insecticide resistance in Ae. aegypti populations in five small towns surrounding the city of Merida, Mexico.
\end{abstract}

Methods: A cross-sectional, entomological survey was performed between June and August 2013 in 250 houses in each of the five towns. Indoor resting adult mosquitoes were collected in all houses and four ovitraps were placed in each study block. CDC bottle bioassays were conducted using $F_{0}-F_{2}$ individuals reared from the ovitraps and $k d r$ allele (lle1016 and Cys1534) frequencies were determined.

Results: High, but varying, levels of resistance to chorpyrifos-ethyl was detected in all study towns, complete susceptibility to bendiocarb in all except one town, and variations in resistance to deltamethrin between towns, ranging from 63-88 \% mortality. Significant associations were detected between deltamethrin resistance and the presence of both $k d r$ alleles. Phenotypic resistance was highly predictive of the presence of both alleles, however, not all mosquitoes containing a mutant allele were phenotypically resistant. An analysis of genotypic differentiation (exact $\mathrm{G}$ test) between the five towns based on the adult female Ae. aegypti collected from inside houses showed highly significant differences $(p<0.0001)$ between genotypes for both loci. When this was further analyzed to look for fine scale differences at the block level within towns, genotypic differentiation was significant for both loci in San Lorenzo (lle1016, $p=0.018$ and Cys1534, $p=0.007$ ) and for lle1016 in Acanceh $(p=0.013)$ and Conkal $(p=0.031)$.

Conclusions: The results from this study suggest that 3 years after switching chemical groups, deltamethrin resistance and a high frequency of $k d r$ alleles persisted in Ae. aegypti populations. The spatial variation that was detected in both resistance phenotypes and genotypes has practical implications, both for vector control operations as well as insecticide resistance management strategies.

Keywords: Aedes aegypti, Dengue, Insecticide resistance, $k d r$

\footnotetext{
*Correspondence: AJL8@cdc.gov

${ }^{4}$ Division of Parasitic Diseases and Malaria, Entomology Branch, Centers for Disease Control and Prevention, Center for Global Health, Atlanta, GA, USA Full list of author information is available at the end of the article
} 


\section{Background}

Dengue is the most important and widespread mosquito-borne viral infection of humans in the world [1]. An estimated 390 million cases of dengue virus (DENV) infection occur per year throughout the tropical and subtropical world. It is estimated that up to $55 \%$ of the world's population is at risk of infection in 128 countries, where 824 million people live in urban environments [1]. In the last 20 years, dengue epidemics have increased in both number and magnitude, due to a range expansion of the Aedes aegypti mosquito, the primary vector of dengue viruses, as well as increased trends in urbanization and global travel and weakened public health infrastructure [2, 3].

In the absence of effective therapeutic medications and vaccines, Ae. aegypti vector control is presently the only approach for preventing and controlling dengue virus transmission $[4,5]$. Vector control strategies rely heavily on the application of chemical insecticides which target immature mosquitoes in their development sites and adult mosquitoes which are often targeted through the use of ultra-low-volume (ULV) and indoor space spraying $[5,6]$. Due to the daytime biting behavior of female Ae.aegypti, the use of bednets as a protective barrier is not generally recommended, although the presence of insecticide treated bednets in houses has been associated with lowered dengue vector infestation indices [7]. Other insecticide-treated materials such as window and door curtains and screens and water storage container covers have also been shown to be effective at reducing household-level Ae. aegypti infestations [8-15], but the impact of such entomological reductions on the risk of dengue virus transmission remains to be determined [16].

As the importance of dengue as a public health problem has increased globally, insecticide-based vector control interventions have been widely employed. The heavy reliance on chemical insecticides to control dengue vectors has led to the development of resistance to many of the insecticides most commonly applied. In the Americas alone, resistance to multiple classes of insecticides has been widely reported in Ae. aegypti [17-23]. This is cause for alarm to those involved in dengue prevention and control, as the growing prevalence of resistance could threaten vector control efficacy [21, 23-25].

Resistance to insecticides can be caused by a variety of physiological changes within a mosquito, including structural alterations at the target site of the insecticide, increased activity of enzymes associated with insecticide detoxification, and changes to the mosquito cuticle. Non-synonymous mutations in the voltage-gated sodium channel transmembrane protein (para) can result in 'knockdown resistance' $(k d r)$, in which the binding of
DDT and pyrethroid insecticides are reduced at this target site [26-29]. There are several point mutations known to confer $k d r$-type insecticide resistance in $A e$. aegypti. In the Americas, substitutions at codons 1016 (V1016I) and 1534 (F1534C) on domains II and III of the voltage gated sodium channel are strongly associated with DDT and pyrethroid resistance in Ae. aegypti [27, 30, 31].

Pyrethroids have become the most frequently used public health insecticides globally due to their low cost and low toxicity to mammals, in addition to their high residual power [32]. It is of considerable concern when $k d r$ is found in wild populations of vector mosquitoes, given there are few suitable alternatives to pyrethroid insecticides approved for public health use. Previous research has demonstrated that $k d r$ mutations arose and spread rapidly in Mexican Ae aegypti populations. Between 2007 and 2009, Siller et al. [33] reported an increase in the frequency of the Ile1016 allele in several localities within the state of Veracruz, Mexico. Similar findings by Ponce Garcia et al. [34] showed over the course of 14 years, several Mexican states, including Veracruz and Yucatan, experienced a significant increase in the frequency of the Ile1016 allele. In the city of Merida, in Yucatan State, Ile1016 had not been detected in 1999 , yet by 2007, it was occurring at frequencies as high as $54 \%$. A more recent study in the state of Guerrero, in southern Mexico, found Ile1016 at a frequency of $80 \%$,with all mosquitoes also containing the Cys1534 allele [35]. The F1534C substitution was initially reported in Ae. aegypti mosquitoes in Asia and first detected in North America in the Cayman Islands [30]. The detection of the Cys1534 allele in Guerrero was the first report of that particular $k d r$ mutation in Mexico [35].

Dengue outbreaks have been reported in Mexico since 1979, and all four DENV serotypes have been reported in Mexico [36]. It is well documented that as viral serotypes are displaced by the introduction of new serotypes, the incidence of severe dengue increases [37, 38]. Yucatan State experienced a similar trend with the incidence of severe dengue increasing each time a new serotype was introduced [12]. The city of Merida is the capital and main urban center of Yucatan State, and dengue transmission in Merida is hyper-endemic. In recent decades, co-circulation of multiple serotypes and a high abundance of Ae.aegypti has increased the risk of severe dengue for the population living in and around the city center [12].

From 1998 to 2009, pyrethroids were the primary insecticides used for adult mosquito control in Mexico, and deltamethrin was the primary insecticide used for indoor space spraying when a dengue case was reported. In 2010, reports of high levels of pyrethroid resistance and a high frequency of the Ile1016 allele in Mexico [34, 
35] prompted vector control authorities to modify their strategy. From 2010 up until when collections for this study were conducted in 2013, carbamates (bendiocarb, and later propoxur) had been predominantly used for indoor space spraying, although the pyrethroids bifenthrin and sumithrin continued to be used at a reduced level.

While the intensity of insecticide use for dengue vector control in the city of Merida is relatively consistent, the surrounding communities have experienced more sporadic insecticide applications. Little entomological data, including resistance data, are available from these surrounding towns. Previous research [34] has suggested a high degree of heterogeneity in insecticide resistance frequency across the Yucatan Peninsula, yet little is known about these patterns of resistance at a finer spatial scale. Spatial heterogeneity of insecticide resistance could have important implications for vector control efficacy, particularly when vector control strategies are designed to be applied across a large geographical area. The development of scientifically sound vector control and insecticide resistance management strategies for Ae. aegypti depends on understanding the patterns and drivers of spatial heterogeneity in insecticide resistance. In the present study, we characterized patterns of
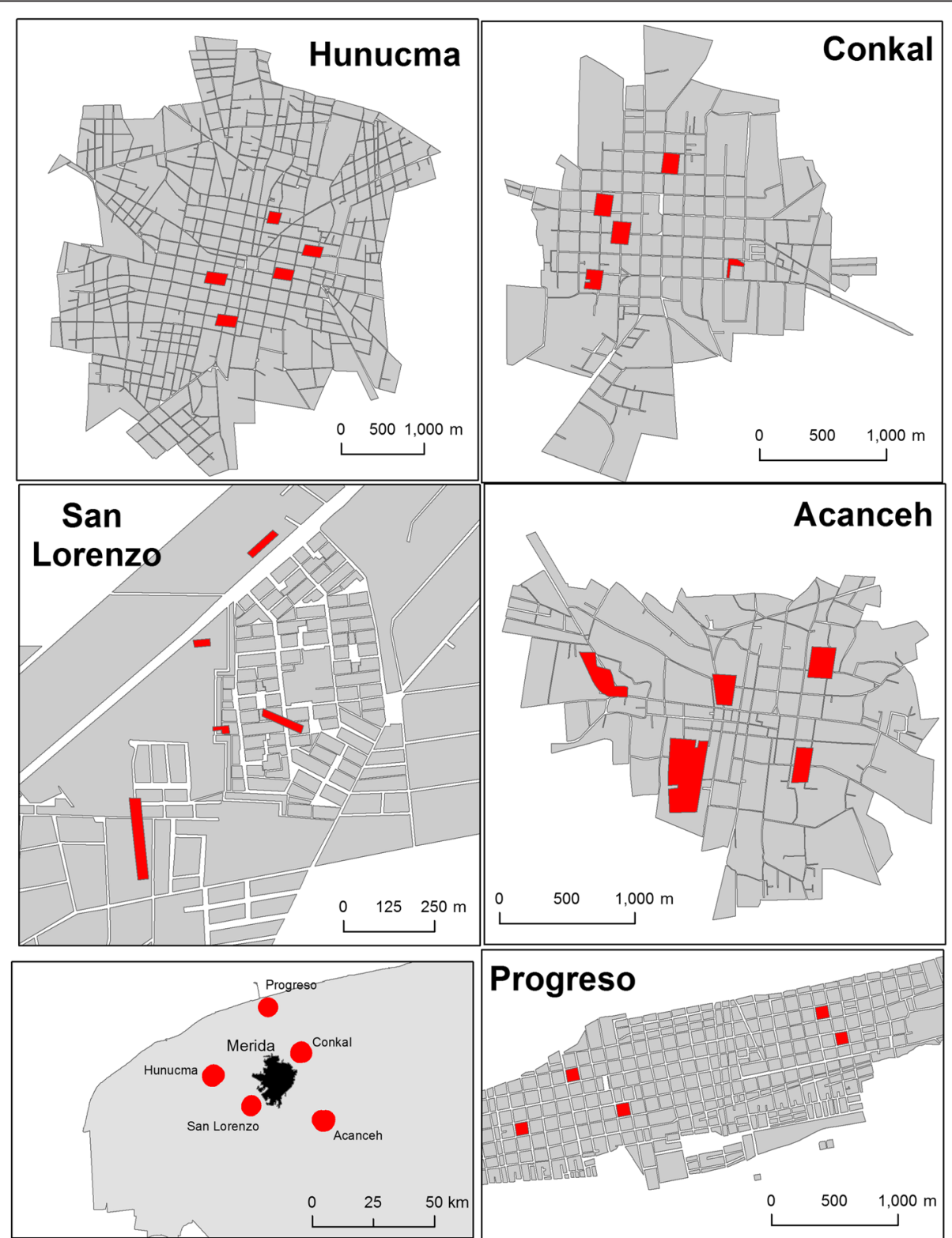

Fig. 1 Maps showing the study areas. Location of Merida, Yucatan, Mexico and the five study towns (lower left panel), with each block sampled in each town highlighted 
insecticide resistance in Ae. aegypti populations within five towns surrounding the city of Merida, Mexico, to gain a greater understanding of how resistance may be distributed at a fine geographical scale.

\section{Methods}

\section{Study area}

This study was conducted in the state of Yucatán in southern Mexico, and included five towns located on the periphery of the state's capital, Merida (population $\sim 1$ million). Merida is located in a subtropical environment with mean temperatures ranging from $29{ }^{\circ} \mathrm{C}$ in December to $34{ }^{\circ} \mathrm{C}$ in July. The rainy season occurs from May to October, which overlaps with the peak dengue transmission season between July and October, although cases occur year-round [39]. Dengue is highly endemic throughout the Yucatan peninsula, and the vector control strategies used at the time of this study included ultra-low volume (ULV) spraying with the organophosphate insecticide chlorpyrifos-ethyl, indoor space spraying with the pyrethroid deltamethrin or the carbamate bendiocarb, and the application of the organophosphate larvicide temephos for breeding site control (Che-Mendoza, Secretaria de Salud de Yucatan, personal communication). Surrounding Merida are small, densely populated, satellite towns that are normally connected to Merida by a single road. The five towns selected for this study were San Lorenzo, Acanceh, Progreso, Hunucma and Conkal. All towns were located 15$35 \mathrm{~km}$ from Merida's city center and at least $20 \mathrm{~km}$ from one another (Fig. 1). Each town has its own municipal jurisdiction, including environmental management entities who engage in breeding site control activities in addition to the vector control interventions conducted by the state-level vector control authorities.

\section{Study design}

A cross-sectional, entomological survey was performed between June and August 2013 in 250 houses across the five satellite towns. A spatially random selection of houses nested in blocks was performed to representatively capture the geography of each town. Briefly, the location of all the urban blocks of each town was digitized using a Geographic Information System (ArcGIS 10.1, ESRI, Redlands CA). This block-level map was then used to group the blocks into $90^{\circ}$ sectors equivalent to the cardinal directions. One block was randomly selected from each one of the sectors (total four blocks) and one additional block was randomly selected from the most central blocks in the town (Fig. 1). On each sampling block, ten houses were randomly selected to perform the entomological collections.

\section{Entomological collections}

Indoor adult mosquito collections were performed between 8:00 am and 2:00 pm by five two-person teams using Prokopack aspirators [40]. Collections were limited to a maximum of 10-15 min per team per household. Each premise was additionally checked for the presence of Ae. aegypti breeding sites. All collected adult mosquitoes were killed by freezing and visually identified to species. All Ae. aegypti females were subsequently desiccated and stored at $-20{ }^{\circ} \mathrm{C}$ for future molecular analysis.

Ae. aegypti ovitraps [41] consisting of 51 dark buckets were placed in the houses located closest to the corners of each block (four traps per block) to collect Ae. aegypti eggs. Ovitrap fabric was checked weekly for a maximum of 3 weeks, and the fabric containing Ae. aegypti eggs was dried and stored in sealed plastic bags until rearing. Eggs were subsequently reared under insectary conditions to provide material for the insecticide resistance bioassays.

\section{Insecticide bioassays}

Eggs collected from the ovitraps in each town were pooled to generate a single, geographically diverse colony for each town. The eggs were hatched and reared to adulthood under constant temperature and humidity conditions at the CDC insectaries (Atlanta, USA). Adult females emerging from the five Ae. aegypti colonies were evaluated for resistance to deltamethrin, bendiocarb and chlorpyrifos-ethyl using the CDC bottle bioassay protocol [42]. Mortality at the CDC-recommended diagnostic doses (DD; deltamethrin: $10 \mu \mathrm{g} /$ bottle, bendiocarb: $12.5 \mu \mathrm{g} /$ bottle, chlorpyrifos: $50 \mu \mathrm{g} /$ bottle) was recorded at the diagnostic time (DT) of $30 \mathrm{~min}$. [42] For each town, 3-4 replicates were conducted for each insecticide, and approximately 20 female Ae. aegypti were used per bottle $($ mean $=20.8$, standard deviation $=1.7)$.

Tests were conducted on $\mathrm{F}_{0}-\mathrm{F}_{2}$ Ae. aegypti. Mosquitoes were classified as phenotypically resistant or susceptible to each insecticide based on their knockdown status at the DT. Individuals were considered to be knocked down if they were no longer able to stand at the diagnostic time. Both resistant and susceptible individuals from the bioassays were subsequently $k d r$-genotyped for Ile1016 and Cys1534.

\section{Molecular assays}

Genomic DNA was extracted from a leg or other body part from each individual adult female mosquito in a solution of $45 \mu \mathrm{l}$ of $\mathrm{H}_{2} \mathrm{O}$ and $5 \mu \mathrm{l}$ of Promega Taq DNA Polymerase10x Buffer with $\mathrm{MgCl}_{2}$ (Madison, WI) in a 96 well PCR plate. Samples were incubated at $95{ }^{\circ} \mathrm{C}$ in a Bio-Rad iCycler ${ }^{\mathrm{Tw}}$ thermal cycler for $15 \mathrm{~min}$. 
Allele-specific PCR was carried out in a Bio-Rad CFX96 Real-Time System C1000 thermal cycler to determine genotype at both loci through analysis of the PCR product melting curves. The PCR reaction to detect the Ile1016 allele was based on the methodology described by Saavedra-Rodriguez et al. [27] and consisted of $4 \mu \mathrm{l}$ of $\mathrm{iQ}^{\mathrm{m}} \mathrm{SYBR}^{\circ}$ Green Supermix (BioRad 170-8880), $2 \mu \mathrm{l}$ of each of the Val1016f, Ile1016f and Ile1016r primers, and $1 \mu \mathrm{l}$ of DNA template. The PCR reaction to detect the Cys1534 allele was based on the methodology described by Yanola et al. [43] and consisted of $7.67 \mu \mathrm{l}$ of $\mathrm{iQ}^{\mathrm{mm}} \mathrm{SYBR}^{\circ}$ Green Supermix (Bio-Rad 170-8880),1 $\mu$ l each of the Phe1534 $+\mathrm{f}$ and Phe1534 $+\mathrm{r}$ primers and $0.33 \mu \mathrm{l}$ of the Cys1534 $+\mathrm{f}$ primers, and $1 \mu \mathrm{l}$ of DNA template [43]. Results were read using Precision Melt Analysis Software ${ }^{\mathrm{Tm}}$. For the $1016 \mathrm{I}$ mutation, a melting peak at $79{ }^{\circ} \mathrm{C}$ corresponds to isoleucine and a melting peak at $85{ }^{\circ} \mathrm{C}$ corresponds to valine (wild type). For the $1534 \mathrm{C} \mathrm{mu-}$ tation, a peak at $85{ }^{\circ} \mathrm{C}$ corresponds to cysteine and a peak at $80{ }^{\circ} \mathrm{C}$ corresponds to phenylalanine (wild type). Genomic DNA from the Rockefeller Ae. aegypti strain was used as a susceptible (wild-type) control. DNA from previously genotyped individuals was used for positive controls for both $k d r$ mutations.

\section{Data analysis}

Per CDC bottle bioassay guidelines, populations were classified as resistant or susceptible using the updated WHO guidelines [44]: 98-100 \% mortality indicates susceptibility, 90-97 \% mortality suggests resistance may be developing, and mortality less than $90 \%$ indicates resistance.

The allele frequencies for Ile1016 and Cys1534 were calculated using the equation:

$$
\frac{\mathrm{n} \text { heterozygotes }+2(\mathrm{n} \text { homozygotes })}{2(\text { total } \mathrm{n} \text { mosquitoes analyzed })}
$$

The $95 \%$ confidence interval (CI95) around the frequency of each of the alleles was calculated using a Wald interval [34]. Fisher's exact tests were performed in SAS 9.3 to test the association between genotype and resistance phenotype. Analysis of linkage disequilibrium was also conducted between sites 1016 and 1534 using Genepop version 4.2 [45].

A genotypic differentiation analysis was performed using Genepop version 4.2 on the adult female Ae. aegypti collected from inside the houses to assess the significance of genotype differences between towns (exact $G$ test) and for genotype differences between blocks within each town [45].

\section{Results}

A total of 545 adult female Ae. aegypti were collected from inside the houses across the five towns (Table 1).

\section{Insecticide bioassays}

CDC bottle bioassays were performed in which 402 female Ae. aegypti were tested for resistance to chlorpyrifos-ethyl, 359 were tested for resistance to bendiocarb, and 429 were tested for resistance to deltamethrin. Resistance to chlorpyrifos-ethyl was observed in all five communities at differing levels, ranging in mortality from $13.8 \%(\mathrm{SE} \pm 8.0)$ in Acanceh to $57.2 \%$ $(\mathrm{SE} \pm 8.7)$ mortality in Hunucma. The development of resistance to bendiocarb was only detected in one of the five communities, Progreso (95\% mortality, SE \pm 3.5 ), while all other communities demonstrated complete susceptibility (100 \% mortality). Resistance to deltamethrin also varied between the communities; San Lorenzo showed the lowest mortality rate at $62.7 \%(\mathrm{SE} \pm 11.6)$ while Conkal showed the highest mortality rate at $88.1 \%$ (SE \pm 5.7 ) (Fig. 2).

\section{Kdr genotyping}

PCR to detect Ile1016 and Cys1534 was performed on 422/429 mosquitoes tested for deltamethrin resistance in the CDC bottle bioassay. Of the 545 adult female $A e$. aegypti collected from the houses, 512 were tested for Ile1016 and 507 were tested for Cys1534. (Tables 2 and 3). From all mosquitoes genotyped for the Ile1016 allele $(n=934), 20.0 \%(n=187)$ were wild-type (susceptible) homozygotes (SS), $36.8 \%(n=344)$ were heterozygotes (SR) and $43.1 \%(n=403)$ were homozygous resistant (RR). From all mosquitoes genotyped for the Cys1534 allele $(n=929), 10.3 \%(n=96)$ were SS, $30.5 \%(n=283)$ SR and $59.2 \%(n=550)$ RR.

From genotyping the mosquitoes used in the deltamethrin bioassays, a highly significant association between genotype at position 1016 and deltamethrin resistance was detected in all five communities: San Lorenzo, Acanceh, Hunucma, and Conkal $(p<0.0001)$, Progreso $(p=0.002)$. A significant association between deltamethrin resistance and genotype at position 1534 was seen in three of the five communities: San Lorenzo $(p=0.012)$ and Acanceh and Hunucma $(p<0.0001)$ (Table 2).

Of the mosquitoes resistant to deltamethrin, $98.8 \%$ $(n=84 / 85)$ were positive for both Ile1016 and Cys1534; 82.4\% (70/85) of those were RR at position 1016 and $97.6 \%(83 / 85)$ were RR at position 1534. Of the mosquitoes homozygous RR at position 1016, approximately half $(49.0 \% ; 70 / 143)$ were resistant to deltamethrin, whereas only a third $(33.6 \%$; 83/247) of the mosquitoes homozygous RR at position 1534 were resistant to deltamethrin. Although there were 140 
Table 1 Adult female Ae. aegypti collected from houses

\begin{tabular}{lcccccc}
\hline Town & No. female Ae. aegypti collected & Median & Q1 & Q2 & Q3 & No. with PCR result for one or both kdr alleles \\
\hline San Lorenzo & 155 & 38 & 13 & 38 & 46 & 141 \\
Acanceh & 100 & 16 & 12 & 16 & 32 & 91 \\
Progreso & 61 & 11 & 8 & 11 & 12 & 60 \\
Hunucma & 124 & 20 & 19 & 20 & 31 & 117 \\
Conkal & 105 & 15 & 8 & 15 & 31 & 103 \\
Total & 545 & & & & & 512
\end{tabular}

Median and quartile values of the number of mosquitoes collected per block are shown. Also shown are the number of house-collected mosquitoes that successfully amplified in the molecular assays to detect lle1016 and Cys1534

mosquitoes homozygous RR for both alleles, only $50.0 \%(n=70 / 140)$ of these were phenotypically resistant to deltamethrin. Interestingly, $97.9 \%(n=140 /$ 143) of Ile1016 RR individuals were also Cys1534 RR homozygotes, but only $56.7 \%(n=140 / 247)$ Cys 1534 RR individuals were also Ile1016 RR homozygotes.

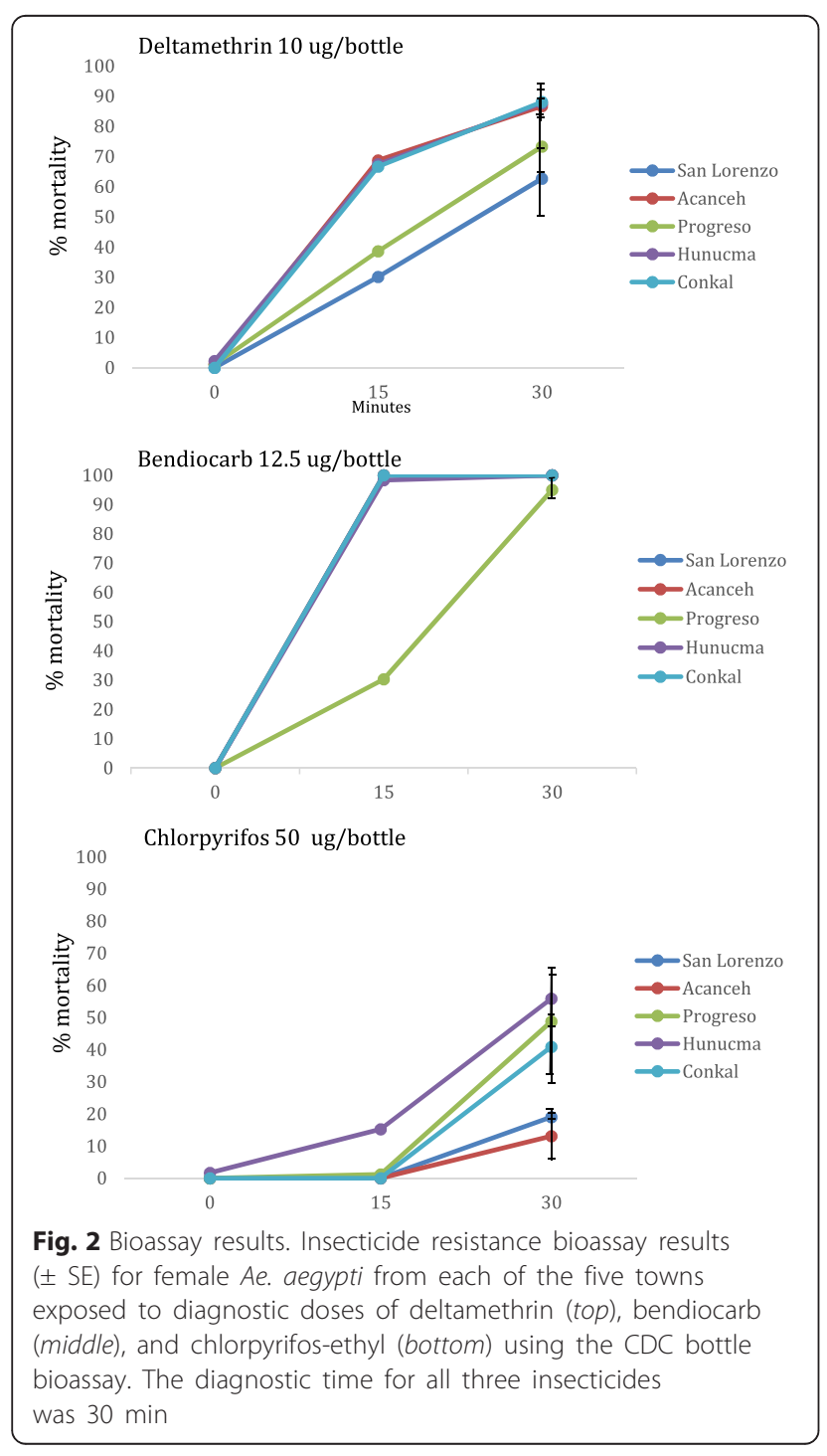

Only three of the Phe1534 SS individuals had anIle1016 allele. As reported in other studies assessing the association between these two resistant alleles $[30,46,47]$, these findings found highly significant linkage disequilibrium between the two loci $(p<0.001)$.

Combining the genotype data from the mosquitoes used in the bioassays and the adult female Ae. aegypti collected from the houses, the overall frequency of Ile1016 across all five towns was $60.6 \%$ (95 \% CI \pm 10.5 ). However, when broken down between towns, the Ile1016 allele frequency varied: Progreso had a frequency of $72.0 \%$ (CI \pm 9.7$)$, San Lorenzo $69.6 \%$ (CI \pm 9.9 ), Acanceh $60.5 \%(\mathrm{CI} \pm 10.5)$, Conkal $59.5 \%(\mathrm{CI} \pm 10.6)$, and Hunucma $47.8 \%(\mathrm{CI} \pm 10.8)$. The overall frequency of the Cys1534 allele across all five towns was $83.0 \%$ (CI \pm 8.1 ) but also demonstrated variation when broken down across sites: Progreso had a frequency of $94.0 \%$ (CI \pm 5.1 ), San Lorenzo had a frequency of $93.4 \%$ (CI \pm 5.3 ), Acanceh $68.0 \%$ (CI \pm 10.0$)$, Conkal $59.8 \%$ (CI $\pm 10.6)$, and Hunucma $57.8 \%(\mathrm{CI} \pm 10.6)$.

To obtain a more focused 'snapshot' of allele frequencies across the towns, genotypes at positions 1016 and 1534 were analyzed separately for the adult female mosquitoes that were collected from the houses (Table 3). The analysis of genotypic differentiation (exact $G$ test) between the five towns based on the adult female $A e$. aegypti collected from inside houses showed highly significant differences $(p<0.0001)$ between genotypes for both loci. When this was further broken down to look for fine scale differences at the block level within towns, genotypic differentiation was significant for both loci in San Lorenzo (Ile1016, $p=0.018$ and Cys1534, $p=0.007$ ) and for Ile1016 in Acanceh $(p=0.013)$ and Conkal $(p=$ $0.031)$.

\section{Discussion}

Our results suggest variability in both resistant phenotypes and genotypes between towns at a small geographical scale, illustrating the complex and focal nature of insecticide resistance. Our results also confirmed the association between two $k d r$ mutations and phenotypic 
Table 2 Summary of data relating deltamethrin resistance phenotype to kdr genotype per town

\begin{tabular}{|c|c|c|c|c|c|c|c|c|c|c|c|c|c|c|}
\hline \multirow[b]{2}{*}{ Town } & \multirow[b]{2}{*}{ Deltamethrin Phenotype } & \multirow[b]{2}{*}{$\mathrm{n}$} & \multicolumn{5}{|c|}{ V1016l } & \multicolumn{5}{|c|}{ F1534C } & \multicolumn{2}{|c|}{ Double homozygotes } \\
\hline & & & VN & $\mathrm{V} / \mathrm{I}$ & I/I & Freq. I & $p$ & $F / F$ & $\mathrm{~F} / \mathrm{C}$ & $\mathrm{C} / \mathrm{C}$ & Freq. C & $p$ & $V N \& F / F$ & $I / I \& C / C$ \\
\hline \multirow[t]{3}{*}{ San Lorenzo } & susceptible & 52 & 0 & 38 & 14 & 0.63 & & 0 & 8 & 44 & 0.92 & & 0 & 13 \\
\hline & resistant & 31 & 1 & 4 & 26 & 0.9 & $<.0001$ & 1 & 0 & 30 & 0.97 & 0.012 & 1 & 26 \\
\hline & Total & 83 & 1 & 42 & 40 & 0.74 & & 1 & 8 & 74 & 0.94 & & 1 & 39 \\
\hline \multirow[t]{3}{*}{ Acanceh } & susceptible & 72 & 19 & 45 & 8 & 0.42 & & 7 & 40 & 25 & 0.63 & & 7 & 8 \\
\hline & resistant & 10 & 0 & 0 & 10 & 1.0 & $<.0001$ & 0 & 0 & 10 & 1.0 & $<.0001$ & 0 & 10 \\
\hline & Total & 82 & 19 & 45 & 18 & 0.49 & & 7 & 40 & 35 & 0.67 & & 7 & 18 \\
\hline \multirow[t]{3}{*}{ Progreso } & susceptible & 66 & 10 & 27 & 29 & 0.64 & & 0 & 8 & 58 & 0.94 & & 0 & 29 \\
\hline & resistant & 24 & 0 & 7 & 17 & 0.85 & 0.0021 & 0 & 0 & 24 & 1.0 & 0.074 & 0 & 17 \\
\hline & Total & 90 & 10 & 34 & 46 & 0.7 & & 0 & 8 & 82 & 0.96 & & 0 & 46 \\
\hline \multirow[t]{3}{*}{ Hunucma } & susceptible & 77 & 18 & 54 & 5 & 0.42 & & 8 & 56 & 13 & 0.53 & & 8 & 5 \\
\hline & resistant & 11 & 0 & 2 & 9 & 0.91 & $<.0001$ & 0 & 0 & 11 & 1.0 & $<.0001$ & 0 & 9 \\
\hline & Total & 88 & 18 & 56 & 14 & 0.48 & & 8 & 56 & 24 & 0.59 & & 8 & 14 \\
\hline \multirow[t]{3}{*}{ Conkal } & susceptible & 70 & 8 & 45 & 17 & 0.56 & & 7 & 39 & 24 & 0.62 & & 4 & 15 \\
\hline & resistant & 9 & 0 & 1 & 8 & 0.94 & $<.0001$ & 0 & 1 & 8 & 0.93 & 0.051 & 0 & 8 \\
\hline & Total & 79 & 8 & 46 & 25 & 0.61 & & 7 & 40 & 32 & 0.66 & & 4 & 23 \\
\hline \multirow[t]{3}{*}{ TOTAL } & susceptible & 337 & 55 & 209 & 73 & & & 22 & 151 & 164 & & & 19 & 70 \\
\hline & resistant & 85 & 1 & 14 & 70 & & & 1 & 1 & 83 & & & 1 & 70 \\
\hline & Total & 422 & 56 & 223 & 143 & & & 23 & 152 & 247 & & & 20 & 140 \\
\hline
\end{tabular}

$n=$ number of individuals tested for $k d r$ genotype from each community; V/V and F/F are SS (homozygous susceptible); V/I and F/C are SR (heterozygotes); I/I and $\mathrm{C} / \mathrm{C}$ are RR (homozygous resistant)

resistance to deltamethrin [30], and detected significant differences in $k d r$ allele frequency at multiple spatial scales.

Low mortality was observed in all populations tested for susceptibility to chlorpyrifos-ethyl, demonstrating high levels of resistance to this organophosphate insecticide across the study area. Although all populations were resistant to chlorpyrifos, a high degree of variability was evident, with Acanceh and San Lorenzo showing the greatest resistance $(<20 \%$ mortality) and the remaining towns showing between 40 and $56 \%$ mortality. Interestingly, susceptibility to the carbamate insecticide bendiocarb was high in all communities except Progreso, where mortality was $95 \%$ (suggesting incipient resistance).

Table 3 Frequency of lle1016 (I) and Cys1534 (C) kdr alleles in indoor resting adult female Ae. aegypti

\begin{tabular}{|c|c|c|c|c|c|c|}
\hline Town & Allele & $n$ & Freq. & $95 \%$ Confidence limits & Genotypic differentiation $p$-value & Hardy-Weinberg $p$-value \\
\hline & & & & & Between blocks within the town & \\
\hline \multirow[t]{2}{*}{ San Lorenzo } & I & 113 & 0.674 & \pm 0.086 & 0.002 & $<0.0001$ \\
\hline & C & 133 & 0.931 & \pm 0.043 & 0.0001 & $<0.0001$ \\
\hline \multirow[t]{2}{*}{ Acanceh } & I & 73 & 0.708 & \pm 0.104 & 0.001 & $<0.0001$ \\
\hline & C & 68 & 0.69 & \pm 0.110 & 0.102 & $<0.0001$ \\
\hline \multirow[t]{2}{*}{ Progreso } & । & 55 & 0.75 & \pm 0.114 & 0.4 & 0.492 \\
\hline & C & 57 & 0.915 & \pm 0.072 & 0.35 & 0.042 \\
\hline \multirow[t]{2}{*}{ Hunumca } & I & 66 & 0.425 & \pm 0.119 & 0.565 & $<0.0001$ \\
\hline & C & 88 & 0.568 & \pm 0.104 & 0.823 & 0.430 \\
\hline \multirow[t]{3}{*}{ Conkal } & । & 74 & 0.586 & \pm 0.112 & 0.005 & $<0.0001$ \\
\hline & C & 158 & 0.563 & \pm 0.104 & 0.092 & 0.0685 \\
\hline & & & & & Between the five towns & \\
\hline \multirow[t]{2}{*}{ Total } & I & 381 & 0.626 & \pm 0.049 & $<0.0001$ & $<0.0001$ \\
\hline & C & 434 & 0.727 & \pm 0.042 & $<0.0001$ & $<0.0001$ \\
\hline
\end{tabular}


That such a high level of resistance to an organophosphate didn't hold true with respect to a carbamate suggests that the mechanisms underlying the resistance are unlikely to be due to ace- 1 target site insensitivity, as this would normally result in cross resistance between both organophosphate and carbamate insecticides [48], nor have ace-1 mutations been widely reported in $A e$. aegypti.

Mortality data from the deltamethrin bioassays also showed variation across the study sites. While all populations were categorized as resistant, the most resistant populations, San Lorenzo and Progreso, had mortalities of 63 and $73 \%$, respectively, while the remaining towns all had mortalities between 87 and $88 \%$. Significant associations were detected between deltamethrin resistance phenotype and the presence of both $k d r$ alleles (Ile1016 and Cys1534). Phenotypic resistance was highly predictive of the presence of both alleles, however, not all mosquitoes containing a mutant allele were phenotypically resistant. This is not entirely unexpected, as both Ile1016 and Cys1534 are largely recessive alleles [27, 30]; indeed, our data supported this as over $90 \%$ of heterozygotes for either allele were phenotypically susceptible to deltamethrin.

The Ile1016 allele is known to be associated with resistance to type I and II pyrethroids (including deltamethrin), as well as DDT [27], while the Cys1534 allele is thought to be associated most strongly with resistance to type I pyrethroids such as permethrin, as well as DDT [30]. However, recent evidence suggests that deltamethrin exposure selects for Cys1534 more rapidly than for Ile1016 [49]. Given that not all mosquitoes that were resistant to deltamethrin in the bioassays were homozygous for the $k d r$ alleles suggests that multiple resistance mechanisms are contributing to the resistant phenotypes, most likely including metabolic mechanisms arising from the overproduction of detoxifying enzymes [50]. The role of the S989P $k d r$ mutation is also of potential interest, as it has recently been associated with deltamethrin resistant phenotypes in Asian populations of Ae. aegypti [51, 52]. Investigation into these additional mechanisms and the role detoxifying enzymes may have on resistance to other insecticides in these populations will help to further explain the resistance patterns observed.

The population-level frequencies of both alleles were estimated from the indoor resting adult Ae. aegypti females collected from houses. Genotypic differentiation was significant between the five communities and was further detected between blocks within the same town in San Lorenzo for both $k d r$ alleles and in Acanceh and Conkal for the Ile1016 allele. These results indicate that even at a fine geographical scale, $k d r$ frequencies can differ significantly. This is particularly interesting in light of population genetics studies that have shown that $A e$. aegypti populations in the Yucatan experience free gene flow within $180 \mathrm{~km}$ [53]. This suggests that the observed differences in $k d r$ genotype are being driven by finescale pressures, such as focal insecticide exposure, as was hypothesized in a recent population genetics study by Saavedra et al. [47]. This could suggest an important role in the household use of insecticides in maintaining pyrethroid pressure, even when vector control programs have switched chemical groups. Indeed, the results from this study suggest that 3 years after the vector control program ceased deltamethrin use, deltamethrin resistance and a high frequency of $k d r$ alleles persisted in the Ae. aegypti population.

Some evidence suggests that pyrethroid resistance has an associated fitness cost resulting in reduced larval development and adult longevity [54], although conflicting data suggest that the Cys1534 allele does not confer fitness costs in Ae. aegypti [55]. Evidence of duplication of the sodium channel gene has recently been reported in Ae aegypti [56], and such an event could maintain both wild-type and mutant alleles in the population, simultaneously conferring resistance and reducing associated fitness costs. Although we currently do not know if this duplication has occurred in Ae. aegypti in Mexico, this warrants further investigation, particularly when discerning why such a high frequency of pyrethroid resistance has been maintained despite a near absence of pyrethroid pressure from vector control authorities during the period leading up to our entomological collections.

While this study presents novel findings regarding the variability of insecticide resistance in space, the interpretation of these results is limited by the cross sectional nature of the study and the small number of insecticides and resistance mechanisms investigated. It will be important to further investigate the patterns and drivers of variability in these communities to assess how insecticide use and other factors can drive resistance patterns and explain fluctuations in both space and time. The observed differences in the frequency of $k d r$ alleles in these populations may have occurred due to pressure from widespread applications of insecticides. A recent study conducted in Martinique found that genetic patterns in Ae. aegypti were driven by insecticide pressure [57]. An alternative explanation is that the alleles were introduced through the immigration of mosquitoes from elsewhere, via containers harboring eggs or adult mosquitoes through other forms of transportation [58-60]. Understanding how these factors contribute to the establishment and maintenance of resistance is key to developing effective resistance management and prevention strategies. 
The variations in both resistance phenotypes and genotypes detected in this study have practical implications for vector control operations. The observation of variability between blocks within the same community suggests that blanket vector control strategies at the provincial or even municipal levels may yield mixed results at best, and further highlights the operational challenge of managing resistance in vector populations with varying resistance profiles at a fine spatial scale. Fluctuations in resistance over time will likely further complicate the landscape, highlighting the need for systematic and routine insecticide resistance surveillance.

\section{Conclusions}

The limited number of suitable insecticides for vector control and the logistical constraints inherent in vector control programs make the small-scale tailoring of vector control interventions particularly challenging, but this is a challenge that must be considered given that insecticide resistance can vary over a small scale. The use of multiple active ingredients through rotations or mixtures has been suggested as an effective way to mitigate or stall the emergence of resistance [61-63], at least in the short term. The findings presented here suggest that large-scale blanket vector control strategies may have limited efficacy given that vector populations can vary widely in their susceptibility to insecticides over a small geographical scale. The development of evidence-based resistance management strategies with respect to dengue vectors should be a priority as dengue continues to increase as a global public health problem.

\section{Competing interests}

The authors declare that they have no competing interests.

\section{Authors' contributions}

AL, GVP and PMS conceived of and designed the study; RD, AMB, EUKC, $A C M$ and $B J$ carried out the fieldwork; RD, KL and LV carried out the labwork; $\mathrm{RD}$ analyzed the data; RD, AL and GVP drafted the manuscript. All authors provided critical input regarding the findings and all authors read and approved the final manuscript.

\section{Acknowledgements}

We are grateful to the people of San Lorenzo, Progreso, Acanceh, Hunucma, and Conkal for allowing us to sample mosquitoes in their homes. Funding for this study was provided to PMS by the Consejo Nacional de Ciencia y Tecnología (CONACYT Mexico), Fondo Sectorial de Investigación en Salud y Seguridad Social (SSA/IMSS/ISSSTE-CONACYT) (SALUD-2011-1-161551). The findings and conclusions in this paper are those of the authors and do not necessarily represent the official position of the Centers for Disease Control and Prevention.

\footnotetext{
Author details

'Department of Environmental Sciences, Emory University, Atlanta, GA, USA. ${ }^{2}$ Unidad Colaborativa para Bioensayos Entomológicos, Campus de Ciencias Biológicas y Agropecuarias, Universidad Autónoma de Yucatán, Mérida, Mexico. ${ }^{3}$ Servicios de Salud de Yucatán, Gobierno del Estado de Yucatán, Mérida, Mexico. ${ }^{4}$ Division of Parasitic Diseases and Malaria, Entomology Branch, Centers for Disease Control and Prevention, Center for Global Health, Atlanta, GA, USA.
}

Received: 9 July 2015 Accepted: 28 January 2016

Published online: 04 February 2016

\section{References}

1. Bhatt S, Gething PW, Brady OJ, Messina JP, Farlow AW, Moyes CL, et al. The global distribution and burden of dengue. Nature. 2013;496(7446):504-7.

2. Gubler DJ, Trent DW. Emergence of epidemic dengue/dengue hemorrhagic fever as a public health problem in the Americas. Infect Agents Dis. 1993; 2(6):383-93.

3. Gubler DJ, Clark GG. Dengue/dengue hemorrhagic fever: the emergence of a global health problem. Emerg Infect Dis. 1995; 1(2):55-7.

4. Webster DP, Farrar J, Rowland-Jones S. Progress towards a dengue vaccine. Lancet Infect Dis. 2009;9(11):678-87.

5. Morrison AC, Zielinski-Gutierrez E, Scott TW, Rosenberg R. Defining challenges and proposing solutions for control of the virus vector Aedes aegypti. PLoS Med. 2008;5(3):e68.

6. Reiter P. Oviposition, dispersal, and survival in Aedes aegypti: implications for the efficacy of control strategies. Vector Borne Zoonotic Dis. 2007;7(2): $261-73$.

7. Lenhart A, Orelus N, Maskill R, Alexander N, Streit T, McCall PJ. Insecticidetreated bednets to control dengue vectors: preliminary evidence from a controlled trial in Haiti. Trop Med Int Health. 2008;13(1):56-67.

8. Kroeger A, Lenhart A, Ochoa M, Villegas E, Levy M, Alexander N, et al. Effective control of dengue vectors with curtains and water container covers treated with insecticide in Mexico and Venezuela: cluster randomised trials. BMJ. 2006;332(7552):1247-52.

9. Rizzo N, Gramajo R, Escobar MC, Arana B, Kroeger A, Manrique-Saide P, et al. Dengue vector management using insecticide treated materials and targeted interventions on productive breeding-sites in Guatemala. BMC Public Health. 2012;12:931.

10. Vanlerberghe V, Villegas E, Oviedo M, Baly A, Lenhart A, McCall PJ, et al. Evaluation of the effectiveness of insecticide treated materials for household level dengue vector control. PLoS Negl Trop Dis. 2011;5(3):e994.

11. N'Guessan R, Darriet F, Doannio JM, Chandre F, Carnevale P. Olyset Net efficacy against pyrethroid resistant Anopheles gambiae and Culex quinquefasciatus after 3 years' field use in Côte d'lvoire. Med Vet Entomol. 2001;15:97-104.

12. Lorono-Pino MA, García-Rejón JE, Machain-Williams C, Gomez-Carro S, Nuñez-Ayala G, Nájera-Vázquez Mdel R, et al. Towards a Casa Segura: a consumer product study of the effect of insecticide-treated curtains on Aedes aegypti and dengue virus infections in the home. Am J Trop Med Hyg. 2013;89(2):385-97.

13. Vanlerberghe $V$, Trongtokit $Y$, Jirarojwatana $S$, Jirarojwatana $R$, Lenhart $A$, Apiwathnasorn C, et al. Coverage-dependent effect of insecticide-treated curtains for dengue control in Thailand. Am J Trop Med Hyg. 2013;89(1):93-8.

14. Manrique-Saide P, Che-Mendoza A, Barrera-Perez M, Guillermo-May G, Herrera-Bojorquez J, Dzul-Manzanilla F, et al. Use of insecticide-treated house screens to reduce infestations of dengue virus vectors, Mexico. Emerg Infect Dis. 2015;21 (2):308-11.

15. Che-Mendoza A, Guillermo-May G, Herrera-Bojórquez J, Barrera-Pérez M, Dzul-Manzanilla F, Gutierrez-Castro C, et al. Long-lasting insecticide-treated house screens and targeted treatment of productive breeding-sites for dengue vector control in Acapulco, Mexico. Trans R Soc Trop Med Hyg. 2015;109(2):106-15.

16. Wilson AL, Dhiman RC, Kitron U, Scott TW, van den Berg H, Lindsay SW. Benefit of insecticide-treated nets, curtains and screening on vector borne diseases, excluding malaria: a systematic review and meta-analysis. PLoS Negl Trop Dis. 2014;8(10):e3228.

17. Alvarez LC, Ponce G, Oviedo M, Lopez B, Flores AE. Resistance to malathion and deltamethrin in Aedes aegypti (Diptera: Culicidae) from western Venezuela. J Med Entomol. 2013;50(5):1031-9.

18. Rodriguez MM, Bisset JA, Fernandez D. Levels of insecticide resistance and resistance mechanisms in Aedes aegypti from some Latin American countries. J Am Mosq Control Assoc. 2007;23(4):420-9.

19. Flores AE, Ponce G, Silva BG, Gutierrez SM, Bobadilla C, Lopez B, et al. Wide spread cross resistance to pyrethroids in Aedes aegypti (Diptera: Culicidae) from Veracruz state Mexico. J Econ Entomol. 2013;106(2):959-69.

20. Bisset JA, Marín R, Rodríguez MM, Severson DW, Ricardo Y, French $L$, et al. Insecticide resistance in two Aedes aegypti (Diptera: Culicidae) strains from Costa Rica. J Med Entomol. 2013;50(2):352-61. 
21. Dusfour I, Thalmensy V, Gaborit P, Issaly J, Carinci R, Girod R. Multiple insecticide resistance in Aedes aegypti (Diptera: Culicidae) populations compromises the effectiveness of dengue vector control in French Guiana. Mem Inst Oswaldo Cruz. 2011;106(3):346-52

22. Marcombe S, Mathieu RB, Pocquet N, Riaz MA, Poupardin R, Sélior S, et al. Insecticide resistance in the dengue vector Aedes aegypti from Martinique: distribution, mechanisms and relations with environmental factors. PLoS One. 2012;7(2):e30989.

23. Fonseca-Gonzalez I, Quiñones ML, Lenhart A, Brogdon WG. Insecticide resistance status of Aedes aegypti (L.) from Colombia. Pest Manag Sci. 2011; 67(4):430-7.

24. Flores AE, Albeldaño WW, Fernandez SI, Badii MH, Loaiza H, Ponce GG, et al. Elevated a-esterase levels associated with permethrin tolerance in Aedes aegypti (L.) from Baja California, Mexico. Pestic Biochem Physiol. 2005;82(1): 66-78.

25. Grisales N, Poupardin R, Gomez S, Fonseca-Gonzalez I, Ranson H, Lenhart A. Temephos resistance in Aedes aegypti in Colombia compromises dengue vector control. PLoS Negl Trop Dis. 2013;7(9):e2438.

26. Milani R. Comportamento mendeliano della resistenza alla azione abbatante del DDT; correlazione tran abbatimento e mortalia in Musca domestica L. Riv Parasitol. 1954:15:513-42.

27. Saavedra-Rodriguez K, Urdaneta-Marquez L, Rajatileka S, Moulton M, Flores $A E$, Fernandez-Salas I, et al. A mutation in the voltage-gated sodium channel gene associated with pyrethroid resistance in Latin American Aedes aegypti. Insect Mol Biol. 2007;16(6):785-98.

28. Martins AJ, Lima JB, Peixoto AA, Valle D. Frequency of Val1016lle mutation in the voltage-gated sodium channel gene of Aedes aegypti Brazilian populations. Trop Med Int Health. 2009;14(11):1351-5.

29. Dong K, Du Y, Rinkevich F, Nomura Y, Xu P, Wang L, et al. Molecular biology of insect sodium channels and pyrethroid resistance. Insect Biochem Mol Biol. 2014;50:1-17.

30. Harris AF, Rajatileka S, Ranson H. Pyrethroid resistance in Aedes aegypti from Grand Cayman. Am J Trop Med Hyg. 2010;83(2):277-84.

31. Hu Z, Du Y, Nomura Y, Dong K. A sodium channel mutation identified in Aedes aegypti selectively reduces cockroach sodium channel sensitivity to type I, but not type II pyrethroids. Insect Biochem Mol Biol. 2011:41(1):9-13.

32. WHO. WHO global malaria programme: world malaria report. Geneva: World Health Organization; 2013.

33. Siller Q, Ponce G, Lozano S, Flores AE. Update on the frequency of lle1016 mutation in voltage-gated sodium channel gene of Aedes aegypti in Mexico. J Am Mosq Control Assoc. 2011;27(4):357-62.

34. Garcia GP, Flores AE, Fernández-Salas I, Saavedra-Rodríguez K, Reyes-Solis G, Lozano-Fuentes $S$, et al. Recent rapid rise of a permethrin knock down resistance allele in Aedes aegypti in Mexico. PLoS Negl Trop Dis. 2009;3(10): e531.

35. Aponte AH, Penilla PR, Dzul-Manzanilla F, Che-Mendoza A, Lopez AD, Solis $F$, et al. The pyrethroid resistance status and mechanisms in Aedes aegypti from the Guerrero state, Mexico. Pestic Biochem Physiol. 2013;107(2):226-34

36. Lorono-Pino MA, Farfán-Ale JA, Zapata-Peraza AL, Rosado-Paredes EP, Flores-Flores LF, García-Rejón JE, et al. Introduction of the American/Asian genotype of dengue 2 virus into the Yucatan State of Mexico. Am J Trop Med Hyg. 2004;71(4):485-92.

37. Rico-Hesse R, Harrison LM, Salas RA, Tovar D, Nisalak A, Ramos C, et al, Origins of dengue type 2 viruses associated with increased pathogenicity in the Americas. Virology. 1997;230(2):244-51.

38. Thu HM, Lowry K, Myint TT, Shwe TN, Han AM, Khin KK, et al. Myanmar dengue outbreak associated with displacement of serotypes 2, 3, and 4 by dengue 1. Emerg Infect Dis. 2004;10(4):593-7.

39. Garcia-Rejon JE, Loroño-Pino MA, Farfán-Ale JA, Flores-Flores LF, LópezUribe MP, Najera-Vazquez Mdel R, et al. Mosquito infestation and dengue virus infection in Aedes aegypti females in schools in Merida, Mexico. Am J Trop Med Hyg. 2011;84(3):489-96.

40. Vazquez-Prokopec GM, Galvin WA, Kelly R, Kitron U. A new, cost-effective, battery-powered aspirator for adult mosquito collections. J Med Entomol. 2009;46(6):1256-9.

41. Lenhart $A E$, Walle $M$, Cedillo $H$, Kroeger A. Building a better ovitrap for detecting Aedes aegypti oviposition. Acta Trop. 2005;96(1):56-9.

42. Brogdon WG, MCAllister JC. Simplification of adult mosquito bioassays through use of time-mortality determinations in glass bottles. J Am Mosq Control Assoc. 1998;14(2):159-64.
43. Yanola J, Somboon P, Walton C, Nachaiwieng W, Somwang P, Prapanthadara LA. High-throughput assays for detection of the F1534C mutation in the voltage-gated sodium channel gene in permethrin-resistant Aedes aegypti and the distribution of this mutation throughout Thailand. Trop Med Int Health. 2011;16(4):501-9.

44. WHO. Test procedures for insecticide resistance monitoring in malaria vector mosquitoes. Geneva: World Health Organization; 2013.

45. Raymond M, Rousset F. GENEPOP (version 4.2); population genetics software for exact tests and ecumenicism. J Heredity. 1995;86:248-9.

46. Linss JG, Brito LP, Garcia GA, Araki AS, Bruno RV, Lima JB, et al. Distribution and dissemination of the Val1016lle and Phe1534Cys Kdr mutations in Aedes aegypti Brazilian natural populations. Parasit Vectors. 2014;7:25.

47. Saavedra-Rodriguez K, Beaty M, Lozano-Fuentes S, Denham S, Garcia-Rejon J, Reyes-Solis G, et al. Local evolution of pyrethroid resistance offsets gene flow among Aedes aegypti collections in Yucatan State, Mexico. Am J Trop Med Hyg. 2015;92(1):201-9.

48. Mutero A, Pralavorio M, Bride JM, Fournier D. Resistance-associated point mutations in insecticide-insensitive acetylcholinesterase. Proc Natl Acad Sci U S A. 1994:91(13):5922-6.

49. Alvarez L, Ponce G, Saavedra-Rodriguez K, Lopez B, Flores AE. Frequency of V1016l and F1534C mutations in the voltage-gated sodium channel gene in Aedes aegypti in Venezuela. Pest Manag Sci. 2014;71(6):863-9. Epub ahead of print.

50. Strode C, Wondji CS, David JP, Hawkes NJ, Lumjuan N, Nelson DR, et al. Genomic analysis of detoxification genes in the mosquito Aedes aegypti. Insect Biochem Mol Biol. 2008:38(1):113-23.

51. Faucon F, Dusfour I, Gaude T, Navratil V, Boyer F, Chandre F, et al. Identifying genomic changes associated with insecticide resistance in the dengue mosquito Aedes aegypti by deep targeted sequencing. Genome Res. 2015;25(9):1347-59.

52. Li C, Kaufman PE, Xue RD, Zhao MH, Wang G, Yan T, et al. Relationship between insecticide resistance and kdr mutations in the dengue vector Aedes aegypti in Southern China. Parasit Vectors. 2015;8:325.

53. Gorrochotegui-Escalante N, Gomez-Machorro C, Lozano-Fuentes S, Fernandez-Salas L, De Lourdes Munoz M, Farfan-Ale JA, et al. Breeding structure of Aedes aegypti populations in Mexico varies by region. Am J Trop Med Hyg. 2002;66(2):213-22.

54. Martins AJ, Ribeiro CD, Bellinato DF, Peixoto AA, Valle D, Lima JB. Effect of insecticide resistance on development, longevity and reproduction of field or laboratory selected Aedes aegypti populations. PLoS One. 2012;7(3): e31889.

55. Plernsub S, Stenhouse SA, Tippawangkosol P, Lumjuan N, Yanola J, Choochote $\mathrm{W}$, et al. Relative developmental and reproductive fitness associated with F1534C homozygous knockdown resistant gene in Aedes aegypti from Thailand. Trop Biomed. 2013;30(4):621-30.

56. Martins AJ, Brito LP, Linss JG, Rivas GB, Machado R, Bruno RV, et al. Evidence for gene duplication in the voltage-gated sodium channel gene of Aedes aegypti. Evol Med Public Health. 2013;2013(1):148-60.

57. Marcombe S, Paris M, Paupy C, Bringuier C, Yebakima A, Chandre F, et al. Insecticide-driven patterns of genetic variation in the dengue vector Aedes aegypti in Martinique Island. PLoS One. 2013;8(10):e77857.

58. Raymond M, Callaghan A, Fort P, Pasteur N. Worldwide migration of amplified insecticide resistance genes in mosquitoes. Nature. 1991; 350(6314):151-3.

59. Huber K, Loan LL, Chantha N, Failloux AB. Human transportation influences Aedes aegypti gene flow in Southeast Asia. Acta Trop. 2004;90(1):23-9.

60. Guagliardo SA, Barboza JL, Morrison AC, Astete H, Vazquez-Prokopec G, Kitron U. Patterns of geographic expansion of Aedes aegypti in the Peruvian Amazon. PLoS Negl Trop Dis. 2014;8(8):e3033.

61. Curtis CF. Theoretical-models of the use of insecticide mixtures for the management of resistance. Bull Entomol Res. 1985;75(2):259-65.

62. Curtis CF, Hill N, Kasim SH. Are there effective resistance management strategies for vectors of human-disease. Biol J Linnean Soc. 1993;48(1):3-18.

63. WHO. In: Programme GM, editor. Global plan for insecticide resistance management in malaria vectors. Geneva: World Health Organization; 2012 